\title{
Microfluidic sorting with high-speed single-layer membrane valves
}

\section{Citation}

Abate, Adam R., Jeremy J. Agresti, and David A. Weitz. 2010. “Microfluidic Sorting with HighSpeed Single-Layer Membrane Valves." Applied Physics Letters96 (20): 203509. https:// doi.org/10.1063/1.3431281.

\section{Permanent link}

http://nrs.harvard.edu/urn-3:HUL.InstRepos:41511257

\section{Terms of Use}

This article was downloaded from Harvard University's DASH repository, and is made available under the terms and conditions applicable to Other Posted Material, as set forth at http:// nrs.harvard.edu/urn-3:HUL.InstRepos:dash.current.terms-of-use\#LAA

\section{Share Your Story}

The Harvard community has made this article openly available.

Please share how this access benefits you. Submit a story.

Accessibility 


\title{
Microfluidic sorting with high-speed single-layer membrane valves
}

\author{
Adam R. Abate, ${ }^{1, a)}$ Jeremy J. Agresti, ${ }^{1,2}$ and David A. Weitz ${ }^{1}$ \\ ${ }^{1}$ Department of Physics, School of Engineering and Applied Sciences, Harvard University, Cambridge, \\ Massachusetts 02138, USA \\ ${ }^{2}$ Fluid Discovery, Emeryville, California 94608, USA
}

(Received 1 February 2010; accepted 8 April 2010; published online 21 May 2010)

\begin{abstract}
Sorting is one of the most important applications of microfluidic devices; however, current sorters place specific requirements on the density, size, and electrical properties of the objects to be sorted, limiting applicability. We present widely applicable microfluidic sorting. We use high-speed single-layer membrane valves to control flows in a bifurcating channel junction, to direct the paths of objects. This allows sorting at hundreds of hertz. Moreover, since the sorting action is mechanical, it is very widely applicable-to drops, particles, and even living cells. () 2010 American Institute of Physics. [doi:10.1063/1.3431281]
\end{abstract}

Microfluidic devices are useful for many applications, including chemical separations, ${ }^{1-3}$ particle synthesis, ${ }^{4-6}$ and the study of individual cells. ${ }^{7-9}$ An essential component is sorting: the ability to extract specific objects from a larger population, for example, extracting individual cells from a population of cells. Sorting in microfluidics can be done in many ways. Particle dispersions can be sorted based on density or size $;^{10,11}$ however, this approach is passive, and the specific requirements of density and size limit applicability. Alternatively, dielectrophoresis can be used very effectively to sort droplets with an electric field. ${ }^{12-15}$ Combined with fluorescence techniques, this approach is useful for a range of biological studies, including for artificial selection assays and directed evolution. ${ }^{15}$ However, dielectrophoresis is only applicable to sorting drops, because it requires a large dielectric contrast with the continuous phase; it cannot be used with cells in suspension, because they are mostly water.

A more general sorting approach is to use mechanical membrane valves. ${ }^{16-19}$ The valves squeeze the channels, increasing their resistance, and thereby diverting flows to sort objects. Since this sorting action is mechanical, it is widely applicable; however, current valve sorters are slow, capable of operating at only a few hertz. To fully realize the potential of microfluidic technologies for sorting, a system is needed that combines the speed of dielectrophoresis with the generality of valves.

In this paper, we introduce sorting with high-speed single-layer membrane valves. We use a membrane valve in combination with an asymmetric channel junction. The asymmetry causes objects to naturally flow into a waste channel unless acted on by the valve. When the valve is actuated the asymmetry is inverted, directing objects into a collection channel. Since only a slight change in channel dimensions is necessary to invert the asymmetry, the sorter is fast, operating at several hundred hertz. Moreover, since the sorting action is mechanical, it is widely applicable, to drops, particles, and even cells.

Our microfluidic sorter is fabricated using softlithography in poly(dimethylsiloxane) (PDMS). ${ }^{20}$ It has a constant channel height of $50 \mu \mathrm{m}$. For the sorting, we use a single-layer membrane valve, which consists of an air cham-

${ }^{a)}$ Electronic mail: aabate@seas.harvard.edu. ber that abuts a fluid channel. ${ }^{21,22}$ To use the valve, we pressurize the air channel which deflects the thin adjoining wall into the fluid channel; this perturbs the streamlines in the fluid channel, which can be used to direct the paths of objects in flow. ${ }^{21}$

To control the valve, we use an electrically activated solenoid (Model LHDA0523112H, The Lee Co., USA). The pressure input of the solenoid is connected to house air pressurized to $2 \mathrm{~atm}$. The solenoid is connected to the membrane valve through a $5 \mathrm{~cm}$ piece of $\mathrm{PE} / 2$ tubing with inner diameter $0.38 \mathrm{~mm}$ (Scientific Commodities, Inc.). The solenoid is controlled by a $3 \mathrm{~V}$ signal. When the solenoid is off, the pressures on both sides of the valve membrane are equal, so that it is undeflected. When it is on, the pressure switches to $2 \mathrm{~atm}$; the air chamber is at a higher pressure, pushing the membrane into the fluid channel. ${ }^{21}$

A practical limit to the sorting speed is the response time of the valve. This is equal to the time for the solenoid to switch plus the time for the valve chamber to pressurize. From high-speed movies, we estimate the solenoid switch time to be $3 \mathrm{~ms}$. After the solenoid switches, a pressure shock wave propagates down the valve at the speed of sound, reaching the membrane in $0.1 \mathrm{~ms}$. The time for the chamber to fully pressurize depends on the rate at which additional air flows in; this is limited by the system's hydrodynamic impedance, which has two components: resistance and capacitance. ${ }^{23}$ The resistance is due to the viscous drag and dissipation of the air as it flows down the tube. The resistance of the system $R_{\text {sys }}=R_{\text {tube }}+R_{\text {valve }}$; since the valve has a much narrower cross section than the tube, $R_{\text {valve }} \gg R_{\text {tube }}$. The capacitance is due to the compressibility of air and compliance of the tubing and PDMS walls, which flex outwards as the shock wave enters. The capacitance of the system $\mathrm{C}_{\text {sys }}$ $=\mathrm{C}_{\text {tube }}+\mathrm{C}_{\mathrm{valv}}$; since the tube has a much larger volume than the valve, $\mathrm{C}_{\text {tube }} \gg \mathrm{C}_{\text {valve }}$. The pressurization time $\mathrm{t}_{\text {on }}$ $\sim \mathrm{R}_{\text {valve }} \mathrm{C}_{\text {tube }}=1 \mathrm{~ms}^{23}$ The measured time is $2.5 \mathrm{~ms}$, indicating that these are the significant factors that limit speed. To sort as fast as possible, it is thus most effective to reduce the resistance and capacitance of the system; this is achieved by using a short, wide valve chamber and stiff, narrow-diameter tubing.

The upper limit to the sorting rate is the velocity at which drops can be flowed into the sorting junction without splitting. Splitting occurs because streamlines pull the drops 

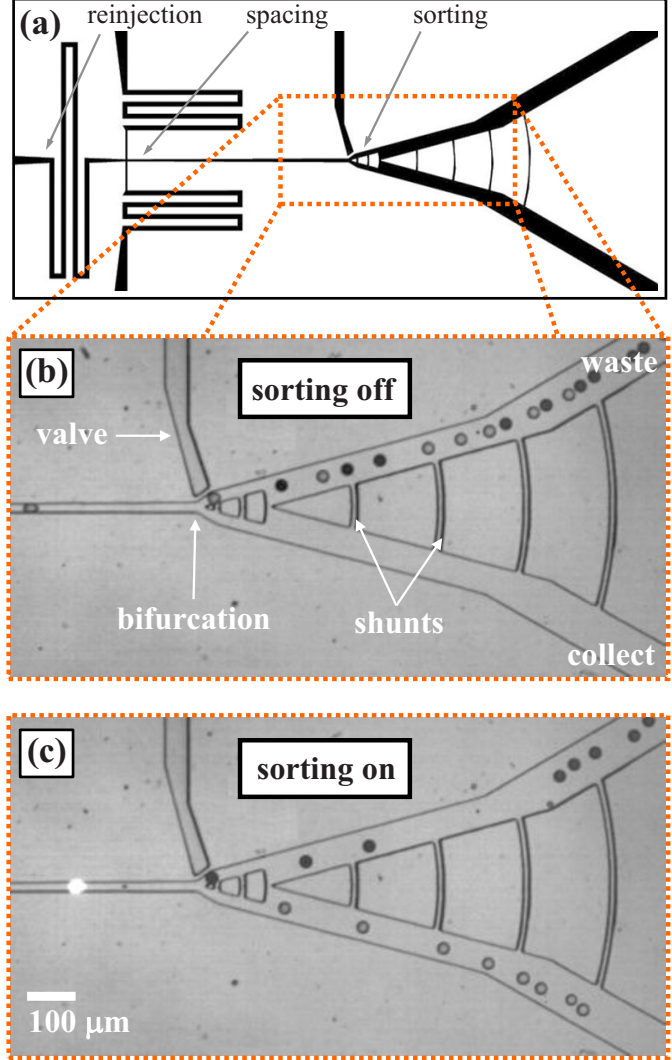

FIG. 1. (Color online) (a) Schematic of valve-based microfluidic sorter. The sorter consists of a spacing device and a sorting device, indicated by the arrows. (b) When the sorting signal is off, all drops flow into the waste channel, due to a slight asymmetry at the bifurcation. (c) When the sorting signal is on, the valve inverts the asymmetry, forcing fluorescent drops in the collection channel.

down both channels; due to viscous drag, this applies an extensional force to the drops. The Capillary number relates the magnitude of viscous forces to surface tension, $\mathrm{Ca}$ $=\mu \nu / \gamma$ where $\mu$ is the viscosity of the liquid, $\nu$ the average velocity, and $\gamma$ the interfacial tension between the two fluid phases. At low $\mathrm{Ca}$, the extensional force is small compared to surface tension, so the drops retain their integrity. However, at high Capillary number, the extensional force exceeds surface tension, tearing drops in two. Precisely where the transition occurs as a function of flow rate depends on channel geometry and fluid properties; ${ }^{24}$ for our system, this occurs at a drop rate of $\sim 10 \mathrm{kHz}$.

Another important feature of our sorter is the use of pressure shunt channels. ${ }^{15}$ These are the small channels connecting the collection and waste channels indicated in Fig. 1(b). Their purpose is to equalize the input pressure after a drop is sorted. Without them, the pressures of both paths would be influenced by drops downstream, making it difficult to sort with the desired control.

To demonstrate how our membrane valve sorter works, we sort a model emulsion. The emulsion consists of light and dark drops; the light drops contain $2 \mathrm{mM}$ fluorescein aqueous and are fluorescent, and the dark drops $5 \mathrm{mM}$ bromphenol blue aqueous and are not fluorescent. For the continuous phase of the emulsion, we use a fluorocarbon oil called HFE7500, with the surfactant ammonium carboxylate of DuPont Krytox 157FSL at $1.8 \mathrm{wt} \%$. The drops for both solutions are made in advance using microfluidic flow focusing; ${ }^{25}$ they are monodisperse, with diameter $28 \mu \mathrm{m}$. The two emulsions are
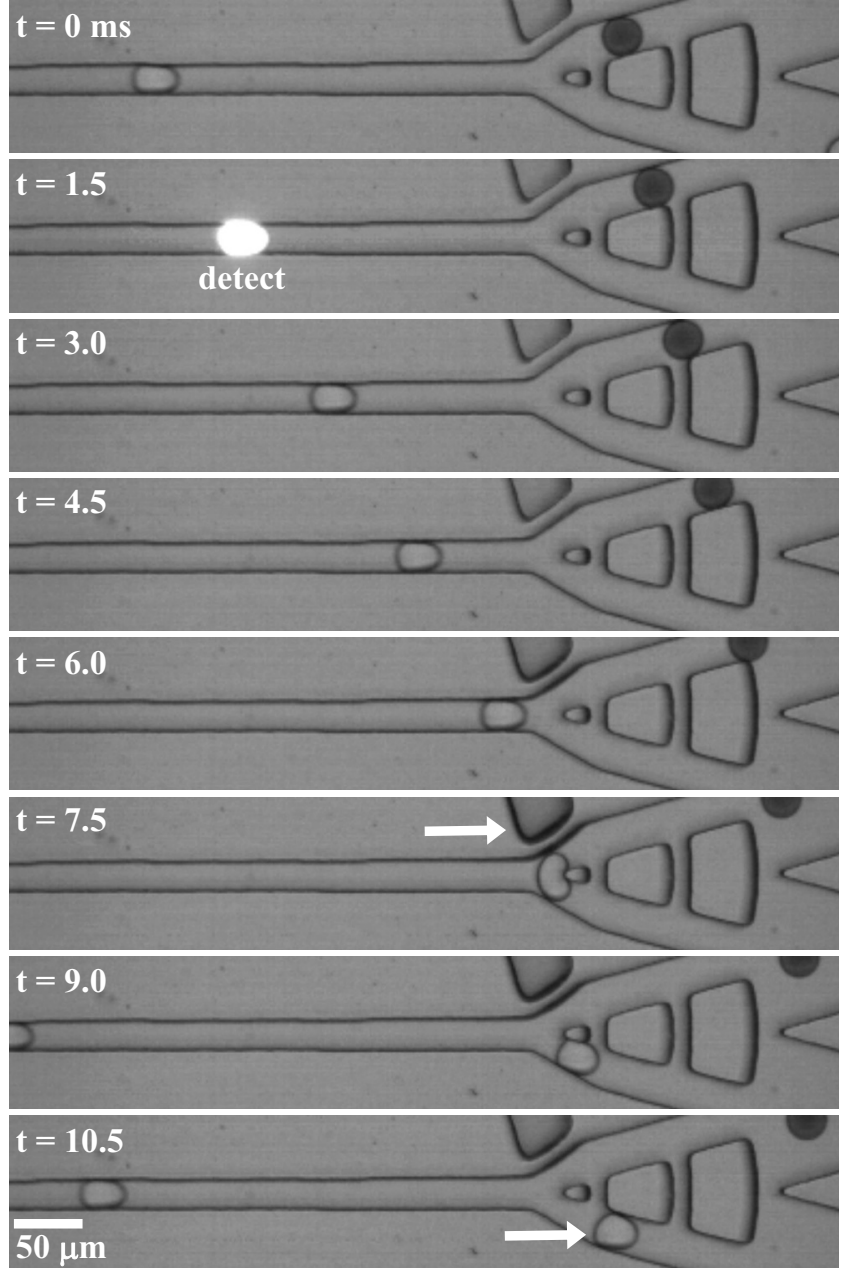

FIG. 2. Example of a drop being sorted. When the fluorescent drop passes through the laser, it emits a burst of light, $\mathrm{t}=3 \mathrm{~ms}$. This triggers actuation of the valve, $\mathrm{t}=9 \mathrm{~ms}$, forcing the drop into the collection channel, $\mathrm{t}=12 \mathrm{~ms}$; nonfluorescent dark drops do not trigger the valve, and thus pass into the waste channel.

combined, producing a random mixture of light and dark drops. This emulsion is reinjected into the sorting device, to sort the light from the dark drops.

The sorter has two following components: a spacing device and a sorting device. The spacing device consists of two channels that intersect at a cross; the reinjected drops enter from a central channel and are spaced by addition of oil from two side channels, as indicated by the arrow in Fig. 1(a). To ensure equal spacing, it is important that the emulsion be reinjected at high droplet-to-oil volume fraction; this causes the drops to enter the spacer close packed and at regular intervals, so they are spaced by equal amounts of oil. Before being sorted, drops are detected by passing them through a laser. The laser excites fluorescence in the drops which is measured using a photomultiplier tube (PMT). The PMT outputs a voltage proportional to the fluorescence intensity. The diameter of the laser spot is smaller than that of the drops, so that the maximum intensity does not depend on drop size. A computer monitors the intensity, actuating the valve when bright drops are detected. The sorting device consists of an asymmetric bifurcating channel and valve. The asymmetry is designed so that all drops to naturally flow upward into the waste channel, as shown in Fig. 1(b). When sorting is turned on and a bright drop is detected, the valve is actuated; this 

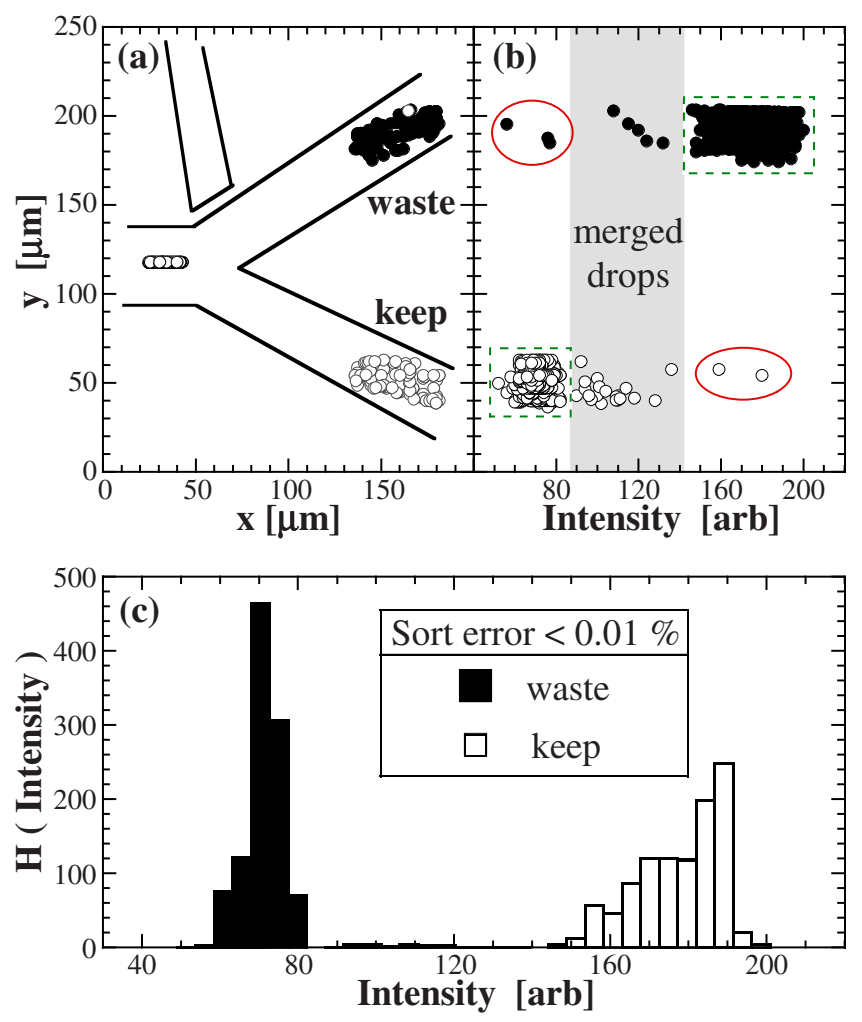

FIG. 3. (Color online) Sorting errors. (a) Vertical and horizontal location after sorting of dark drops (black circles) and light drops (white circles). (b) Vertical location as a function of drop intensity after sorting. Correctly sorted drops are indicated by the dashed boxes, while incorrectly sorted drops are indicated by the solid ovals. All observed sorting errors occur due to the drops in the gray region; these are drops with intermediate intensity, the product of light and dark drops merging. (c) Intensity histograms for drops detected in the waste and collection channels.

inverts the asymmetry, directing drops of interest into the collection channel. Using this simple control of flows, we sort the light from the dark drops, as shown in Fig. 1(c) and in the movies available online. ${ }^{26}$ This allows sorting at a rate of $250 \mathrm{~Hz}$.

To visualize each step in the sorting process, we image one drop being sorted using a high speed camera, Fig. 2. The exposure duration is $100 \mu \mathrm{s}$ and the acquisition rate is 2 $\mathrm{kHz}$. The drop enters the field of view at $\mathrm{t}=0 \mathrm{~ms}$. It is detected as it flows through the laser spot at $\mathrm{t}=1.5 \mathrm{~ms}$. The fluorescent emission triggers the computer, which actuates valve, $\mathrm{t}=6.0 \mathrm{~ms}$. This deflects the drop into the collection channel, as shown at $\mathrm{t}=10.5 \mathrm{~ms}$.

Sorting errors are very rare. To quantify when they do occur, we analyze movies of the sorting process recorded with a high-speed camera. Before sorting, the emulsion consists of a random mixture of light and dark drops. After sorting, the waste channel contains almost entirely dark drops and the collection channel almost entirely light drops, as shown in Fig. 3(a). Occasionally, light drops end up in the waste channel and dark drops in the collection channel, due to sorting errors, as depicted in Fig. 3(a). To reveal the source of this error, we plot drop vertical location versus intensity, after sorting, Fig. 3(b). The vast majority of sorting errors are due to drops in the gray region, having an intermediate intensity. These are the product of two drops of different type merging in the reinjection syringe. Merger can occur due to dust, poor emulsion stability, or wettability defects in the channels. It is very difficult to eliminate, but with care can be mitigated, as demonstrated by our low error rate of $<0.01 \%$ and the nonoverlapping intensity distributions after sorting shown in Fig. 3(c). In the absence of merger, we observe no sorting errors.

Sorting is one of most important applications of microfluidics. Our single-layer membrane valve sorter is simple and robust, while also being very widely applicable, since it is independent of properties such as object density or dielectric constant. This should make it useful for a range of microfluidic sorting applications, including cell and enzyme screens.

We gratefully acknowledge support from the NSF (Grant No. DMR-0602684) and the Harvard MRSEC (Grant No. DMR-0820484).

${ }^{1}$ B. H. Weigl and P. Yager, Science 283, 346 (1999).

${ }^{2}$ B. S. Broyles, S. C. Jacobson, and J. M. Ramsey, Anal. Chem. 75, 2761 (2003).

${ }^{3} \mathrm{H}$. Wu, A. Wheeler, and R. N. Zare, Proc. Natl. Acad. Sci. U.S.A. 101, 12809 (2004).

${ }^{4}$ C.-H. Chen, R. K. Shah, A. R. Abate, and D. A. Weitz, Langmuir 25, 4320 (2009).

${ }^{5}$ Z. Nie, S. Xu, M. Seo, P. C. Lewis, and E. Kumacheva, J. Am. Chem. Soc. 127, 8058 (2005).

${ }^{6}$ H. C. Shum, A. R. Abate, D. Lee, A. R. Studart, B. Wang, C. H. Chen, J. Thiele, R. K. Shah, A. Krummel, and D. A. Weitz, Macromol. Rapid Commun. 31, 108 (2010)

${ }^{7}$ A. R. Wheeler, W. R. Throndset, R. J. Whelan, A. M. Leach, R. N. Zare, Y. H. Liao, K. Farrell, I. D. Manger, and A. Daridon, Anal. Chem. 75, 3581 (2003).

${ }^{8}$ J. El-Ali, P. K. Sorger, and K. F. Jensen, Nature (London) 442, 403 (2006).

${ }^{9}$ C. H. J. Schmitz, A. C. Rowat, S. Köster, and D. A. Weitz, Lab Chip 9, 44 (2009).

${ }^{10}$ M. Yamada and M. Seki, Anal. Chem. 78, 1357 (2006)

${ }^{11}$ D. Huh, J. H. Bahng, Y. Ling, H.-H. Wei, O. D. Kripfgans, J. B. Fowlkes, J. B. Grotberg, and S. Takayama, Anal. Chem. 79, 1369 (2007).

${ }^{12}$ K. Ahn, C. Kerbage, T. P. Hunt, R. M. Westervelt, D. R. Link, and D. A. Weitz, Appl. Phys. Lett. 88, 024104 (2006).

${ }^{13}$ T. P. Hunt, D. Issadore, and R. M. Westervelt, Lab Chip 8, 81 (2008).

${ }^{14}$ J.-C. Baret, O. J. Miller, V. Taly, M. Ryckelynck, A. El-Harrak, L. Frenz, C. Rick, M. L. Samuels, J. B. Hutchison, J. J. Agresti, D. R. Link, D. A. Weitz, and A. D. Griffiths, Lab Chip 9, 1850 (2009).

${ }^{15}$ J. J. Agresti, E. Antipov, A. R. Abate, K. Ahn, A. C. Rowat, J.-C. Baret, M. Marquez, A. M. Klibanov, A. D. Griffiths, and D. A. Weitz, Proc. Natl. Acad. Sci. U.S.A. 107, 4004 (2010).

${ }^{16}$ V. Studer, R. Jameson, E. Pellereau, A. Pépin, and Y. Chen, Microelectron. Eng. 73-74, 852 (2004).

${ }^{17}$ S. Y. Yang, S. K. Hsiung, Y. C. Hung, C. M. Chang, T. L. Liao, and G. B. Lee, Meas. Sci. Technol. 17, 2001 (2006).

${ }^{18}$ S. J. Lee, J. C. Y. Chan, K. J. Maung, E. Rezler, and N. Sundararajan, J. Micromech. Microeng. 17, 843 (2007).

${ }^{19}$ J. Krüger, K. Singh, A. O’Neill, C. Jackson, A. Morrison, and P. O'Brien, J. Micromech. Microeng. 12, 486 (2002).

${ }^{20}$ Y. Xia and G. M. Whitesides, Annu. Rev. Mater. Sci. 28, 153 (1998).

${ }^{21}$ A. R. Abate and D. A. Weitz, Appl. Phys. Lett. 92, 243509 (2008).

${ }^{22}$ A. R. Abate, M. B. Romanowsky, J. J. Agresti, and D. A. Weitz, Appl. Phys. Lett. 94, 023503 (2009).

${ }^{23}$ Wikibooks, Microfluidics/Hydraulic resistance and capacity.

${ }^{24}$ D. R. Link, S. L. Anna, D. A. Weitz, and H. A. Stone, Phys. Rev. Lett. 92, 054503 (2004).

${ }^{25}$ S. L. Anna, N. Bontoux, and H. A. Stone, Appl. Phys. Lett. 82, 364 (2003).

${ }^{26}$ See supplementary material at http://dx.doi.org/10.1063/1.3431281 for videos of the sorting process. 